\title{
Numerical Finiteness Results for Minimal Surfaces in Three-Dimensional Space Forms
}

Ivo Nowak

\section{CONTENTS}

1. Introduction

2. Quasi-Minimal and Minimal Surfaces

3. Examples

4. Numerical Method
Keywords: minimal surfaces, variational principle, finite elements, finiteness results 1991 Mathematics Subject

Classification: 65N30, 53A10, 49Q05, 58E12
We present a new method, based on generalizations of Shiffman's variational principle [Nowak 1993; 1994], for the construction of minimal surfaces on Schwarzian chains in curved space forms. The main emphasis of our approach is on the computation of all minimal surfaces of genus zero (disks with holes) that span a given boundary configuration-even unstable ones. For many boundary configurations we derive numerical finiteness results on the number of minimal surfaces spanning a given boundary configuration. We use graphs of Shiffman's function to illustrate bifurcation phenomena and the Morse index of minimal surfaces. We also present some convergence results for the numerical method.

\section{INTRODUCTION}

Minimal surfaces have long attracted many scientists, partly because of their beauty. Nevertheless, there still remain many open questions. For example, only a few results are known about the number of minimal surfaces spanning a given boundary configuration.

Nowadays soap film experiments are not the only way to improve the understanding of problems concerning minimal surfaces. It is possible to compute minimal surfaces by numerical methods in order to discover new surfaces and to better comprehend known surfaces.

Many numerical methods are based on constructive existence principles, derived in most cases either from nonparametric (geometric measure theoretical) methods or from parametric methods. In contrast, nonparametric methods ignore the conformal structure of minimal surfaces. They have the advantage of being easily applied numerically and of always producing geometrically regular so- 
lutions. The parametric approach, on the other hand, allows the possibility of studying the variation of the conformal structure of minimal surfaces and of describing the set of all minimal surfaces with a prescribed boundary configuration. Therefore, several results about the finiteness of solutions or Morse theory can be proved by parametric methods; see [Jost 1989] and the references there.

Nonparametric numerical methods are described in [Brakke 1992; Chopp and Sethian 1993; Pinkall and Polthier 1993; Hsu et al. 1992], for example. Some of these methods allow the computation of at least some unstable solutions. All of them succeed in very general situations because they do not have to respect the conformal structure of the surfaces.

Parametric numerical methods take into account the conformal structure of the surfaces. Therefore, most of them can treat only simply connected surfaces; see, for example, [Jarausch 1978; Wohlrab 1995; Tsuchiya 1987; Hutchinson 1991; Dziuk and Hutchinson 1996; Hinze 1994]. These methods allow the computation of unstable solutions and in most cases convergence proofs are available.

If $M(c)$ is a three-dimensional space form of curvature $c$, the Schwarzian chain problem in $M(c)$ is the following. Let $\Gamma$ be a Schwarzian chain, that is, a collection of one- or two-dimensional complete geodesic submanifolds of $M(c)$. The problem is to find all minimal surfaces in $M(c)$ whose boundary lies in $\Gamma$ and that intersect the two-dimensional segments of $\Gamma$ perpendicularly.

In this case it is possible to simplify the problem of finding all minimal surfaces with the given boundary configuration by the quasi-minimal surface approach. This approach was originally developed by Courant for the study of unstable disktype polygonal minimal surfaces (simply connected surfaces whose boundary consists of straight lines), and was generalized in [Nowak 1993] for partially free boundaries $M(c)$, under the assumption that the surface lies in a hemisphere if $c>0$. The quasiminimal surface approach is more intricate than a direct variational approach, but it has the advantage that one has only to study a finite-dimensional function, the Shiffman function, for finiteness questions. This is because there is a one-to-one correspondence between the critical points of the Shiffman function and the critical points of Dirichlet's integral.

We developed C software [Nowak 1994] for the computation of minimal surfaces based on the variational principle of [Nowak 1993]. Our approach has several advantages:

- If the boundary configuration does not consists of too many segments, it is possible to determine all minimal surfaces of genus zero on the given boundaries by evaluating Shiffman's function. This gives the possibility of finding new solutions. Figure 11 on page 310 shows a new minimal surface in $\mathbb{R}^{3}$ found using our method.

- The determination of the Morse index of a minimal surface, which is normally a nontrivial problem, can be done by simply evaluating the eigenvalues of the Hessian of Shiffman's function.

- Graphs of Shiffman's function help illustrate bifurcation phenomena that might occur by deforming the boundary.

- In several cases one can prove numerical convergence.

- Quasi-minimal surfaces can be computed very fast. In the Euclidean case it is sufficient to solve only one linear equation.

In Section 3 we present some examples of minimal surfaces computed by our method. Graphs of the Shiffman function indicate that the class of functions with prescribed boundary conditions contains only few critical points of Dirichlet's integral. We also show that the number of critical points in a function class may change if the boundary contour is deformed. We found among other things a very simple boundary contour whose corresponding function class can contain two, one, or zero critical points. It would be desirable to find a formal proof of the finiteness results that we have derived numerically. 


\section{QUASI-MINIMAL AND MINIMAL SURFACES}

\section{Statement of the Problem}

Let $M(c)$ denote the space form of constant curvature $c$. We work with the standard descriptions: $M(0)=\mathbb{R}^{3}$ and, for $c \neq 0$,

$$
M(c)=\left\{X \in \mathbb{R}^{4}:\langle X, X\rangle_{c}^{2}=1 / c\right\},
$$

where $\langle a, b\rangle_{c}$ is the Euclidean scalar product if $c$ is positive and the Lorentzian scalar product defined by $-a_{1} b_{1}+a_{2} b_{2}+a_{3} b_{3}+a_{4} b_{4}$ if $c$ is negative.

A Schwarzian chain $\Gamma$ is defined by $\Gamma:=\bigcup_{i=1}^{m} \Gamma_{i}$, where each $\Gamma_{i}$ is a one- or two-dimensional complete geodesic submanifold of $M(c)$. We do not require the union to be disjoint. We propose to solve the Schwarzian Chain Problem, which is this:

Problem A. Given a Schwarzian chain $\Gamma$ in $M(c)$, find all minimal surfaces in $M(c)$ whose boundary is contained in $\Gamma$ and that are perpendicular to the two-dimensional components of $\Gamma$.

Minimal surfaces defined in the usual sense (monotonicity at the one-dimensional boundary arcs and geometrically meaningful free boundary conditions) are clearly also solutions of the Schwarzian Chain Problem. Therefore, it is not a restriction to consider the Schwarzian Chain Problem.

\section{Parametric Minimal Surfaces}

Jost [1991] showed that any (possibly multiply connected) minimal surface of genus zero $M \subset M(c)$ with nonempty boundaries on a Schwarzian chain $\Gamma:=\bigcup_{i=1}^{m} \Gamma_{i}$ in $M(c)$ can be parametrized conformally by a map $X: \Omega \rightarrow M(c)$, where $\Omega$ is a plane domain bounded by $p$ circles, called a circular domain. $X$ and $\Omega$ can be normalized by a three-point condition, namely, three chosen points on one of the boundary curves of $M$ can be made to correspond to three given points on the outer boundary of $\Omega$, which is taken as the unit circle. A parametrization $X: \Omega \rightarrow M$ of a minimal surface $M$ satisfies five conditions:

(i) $X$ satisfies the boundary conditions. There exist a partition $\left\{\gamma_{i}\right\}_{1 \leq i \leq l}$ of $\partial \Omega$ and an index map
$I:\{1, \ldots, l\} \rightarrow\{1, \ldots, m\}$ such that $\bigcup_{i=1}^{l} \bar{\gamma}_{i}=$ $\partial \Omega, \gamma_{i} \cap \gamma_{j}=\varnothing$ for $i \neq j$ and $X\left(\gamma_{i}\right) \subset \Gamma_{I(i)}$ for $1 \leq i \leq l$. The number $l$ of boundary segments that are mapped into a given Schwarzian chain can be greater than the number $m$ of Schwarzian chain segments because a surface can meet a two-dimensional Schwarzian chain segment several times.

(ii) $X$ is harmonic. It satisfies

$$
X_{z \bar{z}}+c\left\langle X_{z}, X_{\bar{z}}\right\rangle_{c} X=0
$$

on $\Omega$, where $X_{z}=X_{u}+i X_{v}$ is the complex derivative of $X(u, v)$.

(iii) $M$ is parametrized conformally, that is, $X_{z}^{2}=0$ on $\Omega$, where

$$
X_{z}^{2}:=\left|X_{u}\right|_{c}^{2}-\left|X_{v}\right|_{c}^{2}-2 i\left\langle X_{u}, X_{v}\right\rangle_{c} .
$$

(iv) $X$ meets the two-dimensional Schwarzian chain segments perpendicularly:

$$
\left\langle v, X_{n}\right\rangle_{c}=0
$$

for $v \in T_{X(z)} \Gamma_{I(i)}$ and $z \in \gamma_{i}$ if $\Gamma_{I(i)}$ is twodimensional; here $X_{n}$ denotes the derivative in normal direction on $\partial \Omega$, and $T_{p} M$ denotes the tangent space of $M$ at $p \in M$.

(v) $X$ is as regular as $M$. It satisfies

$$
X \in C^{0}(\bar{\Omega}) \cap C^{1}(\bar{\Omega} \backslash V) \cap C^{2}(\Omega),
$$

where $V=\left\{\bar{\gamma}_{i} \cap \bar{\gamma}_{j}: 1 \leq i, j \leq l, i \neq j\right\}$.

\section{Quasi-Minimal Surfaces}

It can be shown by standard variational techniques that a parametrized minimal surface that satisfies the conditions (i)-(iv) of page 303 is a critical point of Dirichlet's integral defined by

$$
D_{\Omega}(X):=\frac{1}{2} \int_{\Omega}\left(\left|X_{u}\right|_{c}^{2}+\left|X_{v}\right|_{c}^{2}\right) d u d v
$$

in a suitable function space. A direct variational approach for constructing minimal surfaces therefore consists in the determination of critical points of Dirichlet's integral. In the case of disk-type 
polygonal surfaces Courant reduced this infinitedimensional variational problem to a finite-dimensional one by the quasi-minimal surface approach, which we will explain shortly. Shiffman later modified Courant's approach by dropping the monotonicity condition at the boundary and by defining the Schwarzian chain by means of complete geodesic submanifolds. (The boundary curve of a surface is allowed to overshoot at the vertices.)

Shiffman's approach was generalized in [Nowak 1993] to the situation that we consider here. This variational principle is the basis for our numerical method.

Let $\Omega$ be a circular domain as defined above, let $\left\{\gamma_{i}\right\}_{1 \leq i \leq l}$ be a partition of $\partial \Omega$, and let $V$ be the set of points $\bar{\gamma}_{i} \cap \bar{\gamma}_{j}$, with $i \neq j$. Each point $z \in V$ can be represented by

$$
z=r_{k} e^{i \tau_{k j}}+m_{k},
$$

with $1 \leq k \leq p, 1 \leq j \leq q_{k}$, and $0 \leq \tau_{k j}<2 \pi$. Here $q_{k}$ is the number of subarcs, $r_{k}$ is the radius and $m_{k}$ is the center of the $k$-th circle. We introduce the parameter

$$
s=\left(r_{1}, m_{1}, \ldots, r_{p}, m_{p}, \tau_{11}, \ldots, \tau_{p q_{p}}\right)
$$

and denote by $\Omega_{s}$ the corresponding circular domain and by $\gamma_{i}^{s}$ the subarcs of the boundary partition; see Figure 1, right. The set $\mathcal{U}$ of admissible

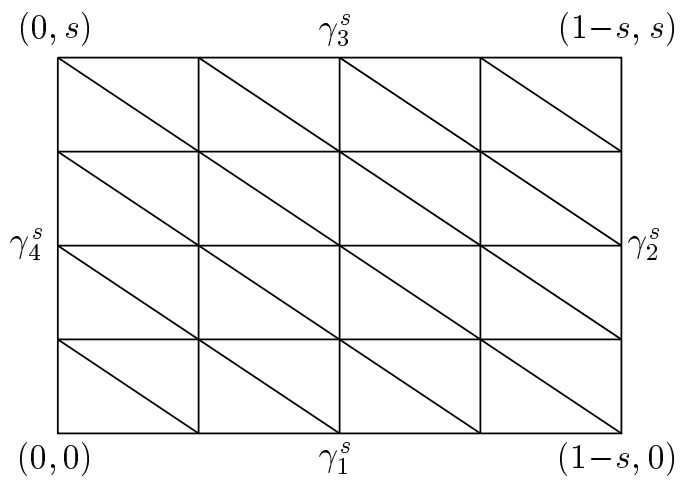

parameters $s$ is defined so that the circles do not intersect or touch and so that a three-point condition is satisfied.

Let $H^{k}\left(\Omega, \mathbb{R}^{n}\right)$ be the Sobolev space of $L^{2}$-functions $X: \Omega \rightarrow \mathbb{R}^{n}$ with square integrable distributional derivatives up to the order $k$ and let

$$
\begin{aligned}
\|X\|_{k, \Omega} & :=\left(\sum_{i=0}^{k}|X|_{i, \Omega}^{2}\right)^{1 / 2}, \\
|X|_{k, \Omega} & :=\left(\int_{\Omega}\left|D^{k} X\right|^{2} d w\right)^{1 / 2} .
\end{aligned}
$$

Let $\Gamma$ be a Schwarzian chain and $I$ be an index map that prescribes the boundary conditions. Shiffman's function space

$$
\mathcal{C}(\Gamma, I, s)
$$

is defined for $s \in \mathcal{U}$ as the set of $Y \in H^{1}\left(\Omega_{s}\right)$ such that $Y\left(\Omega_{s}\right) \subset M(c)$ almost everywhere and $Y\left(\gamma_{i}^{s}\right) \subset \Gamma_{I(i)}$ almost everywhere, for $1 \leq i \leq l$.

The key in applying Shiffman's variational principle to the non-Euclidean case is a uniqueness result for critical points of Dirichlets's integral in Shiffman's function space. Ströhmer [1980] did this for polygonal boundaries in nonpositively curved space forms. A straightforward generalization to positively curved space forms is not possible, since a geodesic between two given points is not unique

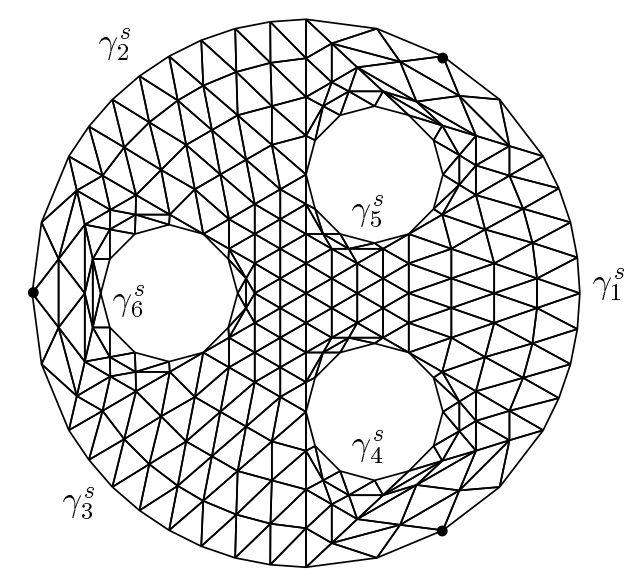

FIGURE 1. Triangulation of a simply connected and a multiply connected parameter domain. 
in this case. Therefore, we introduce the following condition:

Condition 1. If $c>0$ we assume that the Schwarzian chain $\Gamma$ is contained in a hemisphere of $M(c)$; that is, there exists a vector $v \in \mathbb{R}^{4}$ such that $\langle x, v\rangle>0$ for all $x \in \Gamma$.

The assumption implies that in the case of positively curved space forms we consider only those minimal surfaces that are contained in a hemisphere of $M(c)$. The Schwarzian chain does not consist of complete geodesics in this case.

To guarantee the existence and nondegeneracy of surfaces we introduce the following condition:

Condition 2. (i) For all $s \in \mathcal{U}$, the function space $\mathcal{C}(\Gamma, I, s)$ is nonempty and does not contain contant functions.

(ii) $\bigcap_{i=1}^{l} T \Gamma_{I(i)}=\{0\}$, where $T M:=\bigcup_{p \in M} T_{p} M$.

$\mathcal{C}(\Gamma, I, s)$ is nonempty if the circles of $\partial \Omega_{s}$ can be mapped onto rectifiable closed curves $\sigma$ such that $\sigma\left(\gamma_{i}^{s}\right) \subset \Gamma_{I(i)}$ for $i=1, \ldots, l$. Condition (ii) is a geometrical assumption that means that the intersection of tangent spaces with respect to Schwarzian chain segments is $\{0\}$.

In [Nowak 1993; 1994] we proved that a unique critical point (a minimizer) of Dirichlet's integral exists in the space $\mathrm{e}(\Gamma, I, s)$ if Conditions 1 and 2 are satisfied. This critical point is denoted by $X(s)$ and is called a quasi-minimal surface.

We call $D(s):=D_{\Omega_{s}}(X(s))$ the Shiffman function and $C(s):=D(s)-A_{\Omega_{s}}(X(s))$ the conformal energy, where $A_{\Omega}(\cdot)$ denotes the area functional.

To give rise to a minimal surface, the parameter $s$ has to be chosen in such a way that the corresponding quasi-minimal surface $X(s)$ is parametrized conformally; that is, $X_{z}^{2}(s)=0$ if and only if $C(s)=0$. A parameter $s^{*}$ with this property is called optimal. It is well known that $C(s) \geq 0$. Therefore, optimal parameters can be obtained by minimizing $C(s)$.

Until now it has not been proved that conformal quasi-minimal surfaces satisfy the boundary conditions in the usual way. The surfaces might overshoot at the vertices. We therefore call conformal quasi-minimal surfaces $X\left(s^{*}\right)$ Shiffmanian minimal surfaces. They can be constructed by the following variational principle:

Problem B. Minimize $D_{\Omega_{s}}(Y)$ subject to the condition $Y \in \mathcal{C}(\Gamma, I, s)$, where $s \in \mathcal{U}$ minimizes $C(s)$.

Since parametrized solutions of Problem A that satisfy conditions (i)-(v) of page 303 are critical points of Dirichlet's integral, they are also solutions of Problem B. It can, however, not be guaranteed that solutions of Problem B are physically meaningful; see Figure 14, left.

Remark 1. For numerical reasons sometimes it is more convenient to use other parameter domains than circular ones. The simply connected surfaces of Section 3 were parametrized by a rectangle with edge-sizes $s$ and $1-s, s \in(0,1)$; see Figure 1, left.

Remark 2. Instead of defining an optimal parameter $s^{*}$ by minimizing $C(s)$, it is also possible to define $s^{*}$ by $\nabla D\left(s^{*}\right)=0$. By variations of the parameter domain $\Omega_{s}$ one can show that this is equivalent to $X_{z}^{2}\left(s^{*}\right)=0$ using the formula

$$
\langle\nabla D(s), v\rangle=-\frac{1}{2} \int_{\Omega_{s}} \operatorname{Re}\left(X_{z}^{2}(s) \cdot \tau_{\bar{z}}\right) d z .
$$

Here, $\tau$ is an inner variation of $\Omega_{s}$ depending on $v \in \mathbb{R}^{q}$ (where $s \in \mathbb{R}^{q}$ ) defined by $\tau=\left.(\partial / \partial \varepsilon) g_{\varepsilon}\right|_{\varepsilon=0}$ and $g_{\varepsilon}: \Omega_{s} \rightarrow \Omega_{s+\varepsilon v}$ is a diffeomorphism depending differentiably on $\varepsilon$. If $\Omega_{s}$ is a disk it is easy to calculate $\tau$. But for other domains, such as rectangular or multiply connected ones, the calculation of $\tau$ is a difficult task. Therefore, it is easier to minimize $C(s)$ in numerical algorithms.

Remark 3. Another approach for the construction of unstable minimal surfaces is to minimize Dirichlet's functional subject to a prescribed-volume constraint. The minimum of this volume-constrained variational problem is an $H$-surface, where the constant mean curvature $H$ is the Lagrange parameter corresponding to the volume constraint [Wente 1971]. 
Under certain assumptions unstable minimal surfaces are stable in the volume-restricted function space. Therefore, all minimal surfaces with given boundary can be constructed in these cases by energy minimization methods; see [Brakke 1992], for example.

This principle should be applicable for the construction of large solutions in $S^{3}$. These solutions are not contained in a hemisphere of $S^{3}$, so our Condition 1 is violated in this case.

\section{Stability of Minimal Surfaces}

The stability of a minimal surface can be examined by determining the Morse index, which is, in general, a nontrivial problem. In the case of disktype polygonal minimal surfaces Sauvigny proved [1985b] that the Morse index of a disk-type polygonal minimal surface in $\mathbb{R}^{n}$ is equivalent to the Morse index of Shiffman's function $D(s)$, that is the number of negative eigenvalues of the Hessian $\nabla^{2} D(s)$. A disk-type polygonal minimal surface is therefore unstable if and only if the Hessian $\nabla^{2} D(s)$ has at least a negative eigenvalue.

The numerical examples of Section 3 show that Shiffman's function in the non-Euclidean case is similar to the Euclidean case. For example, the indices of the surfaces in Figures 2 and 4 are known in the Euclidean case and numerical evaluation of the corresponding Shiffman functions demonstrate that they do not change in the non-Euclidean case. Therefore, it can be conjectured that the result of

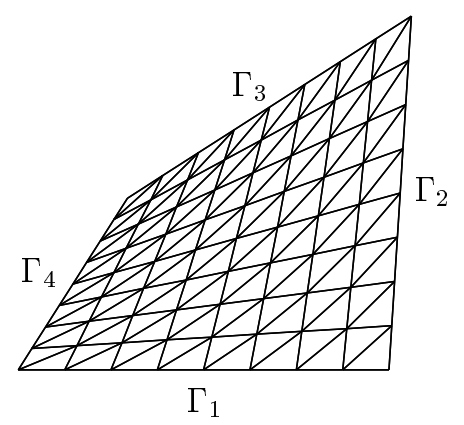

Sauvigny can be generalized to the situation considered here. One difficulty in generalizing Sauvigny's proof is that asymptotic expansions at the vertices of quasi-minimal surfaces are required, and such expansions have only been derived for disktype polygonal Euclidean surfaces [Heinz 1979].

\section{EXAMPLES}

Minimal surfaces on Schwarzian chains have been known for more than 150 years. In this section we shall give examples to demonstrate the complexity of the minimal surface problem that can be observed already when the parameter $s$ is oneor two-dimensional. All graphics were computed by the numerical method described in Section 4 . Simply connected surfaces were parametrized by a rectangle (Figure 1, left), and multiply connected ones by a circular domain (Figure 1, right).

\section{Polygonal Minimal Surfaces}

We begin with some polygonal examples. For disktype polygonal surfaces there exist some uniqueness results, such as for boundaries with a oneto-one projection on a planar convex curve [Radó 1930] or for extreme polygons with total curvature less than $4 \pi$ [Sauvigny 1985a]. For disk-type polygonal surfaces it is also possible to compute the Morse index of a minimal surface by simply computing the Morse index of Shiffman's function [Sauvigny 1985b]. Figure 2, left, shows a polygonal

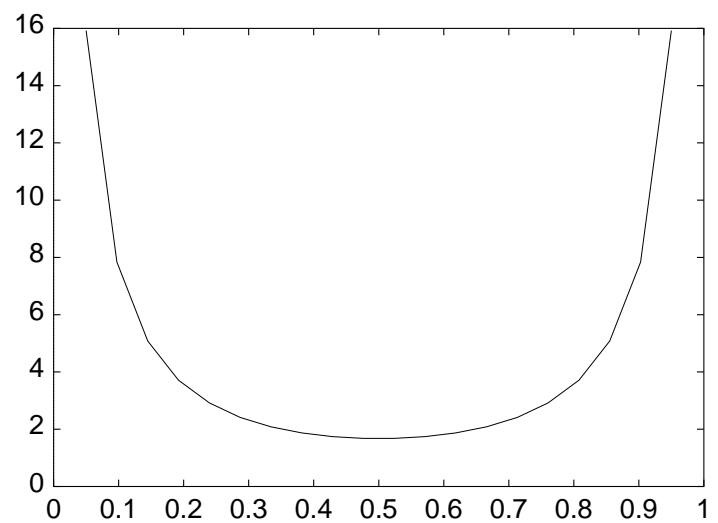

FIGURE 2. Riemann-Schwarz surface and the corresponding Shiffman function. 

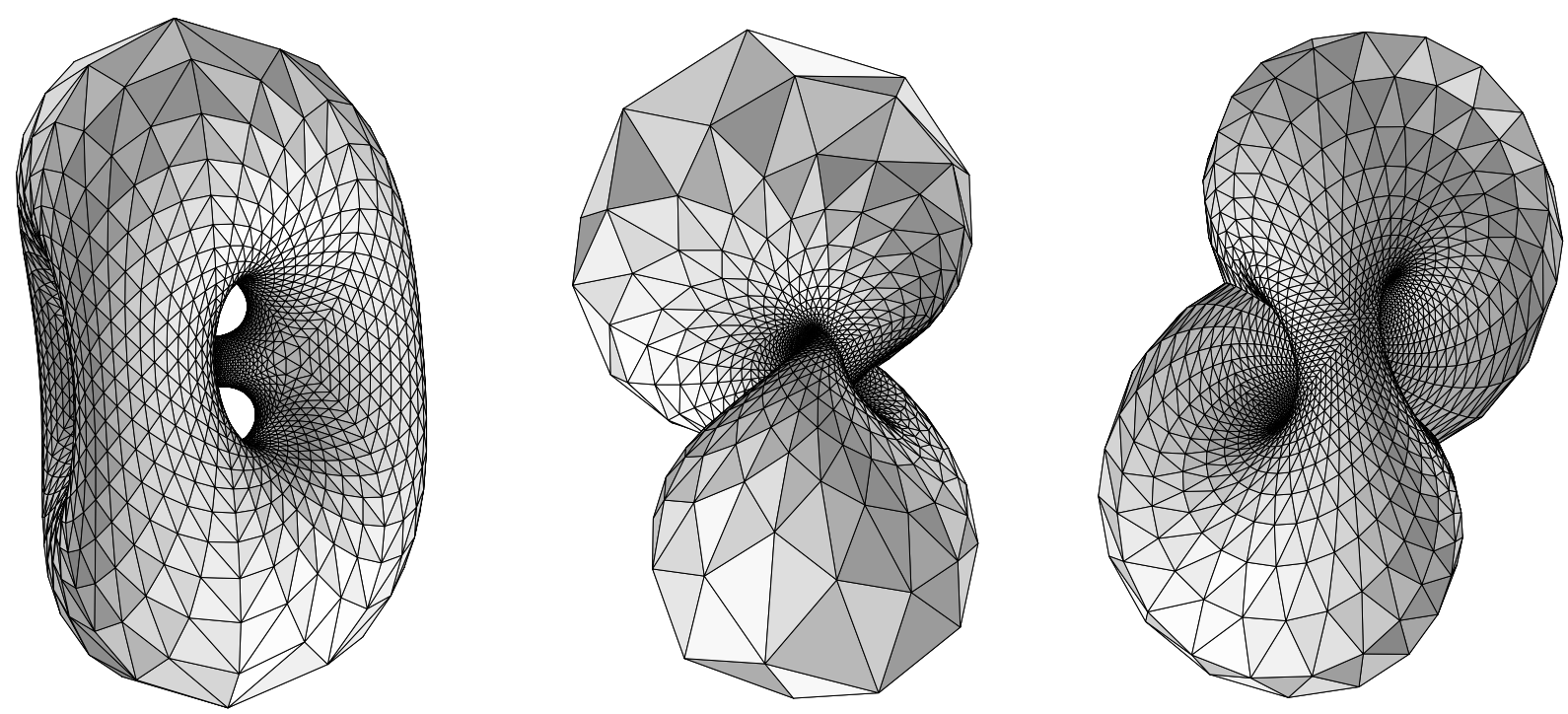

FIGURE 3. Lawson surfaces $\xi_{2,2}, \tau_{2,1}$, and $\eta_{1,1}$ in $S^{3}$, shown in stereographic projection.

minimal surface with four vertices. The parameter $s$ is in this case one-dimensional since three vertices are fixed. The corresponding Shiffman function, shown in Figure 2, right, indicates that the solution is unique and stable.

For some polygonal Schwarzian chains with four vertices and angles of the form $\pi / k$, for $k \in \mathbb{N}$, it is possible to construct complete periodic minimal surfaces in $M(c)$ by multiple reflections of one minimal surface patch. (The minimal surface patch and the Shiffman function look always similar as those of Figure 2.) With the aid of this method Lawson [1970] constructed complete minimal surfaces in $S^{3}$ of arbitrary genus. Figure 3 shows three such examples, the last of which is nonorientable. The generating patches were computed by the numerical method described in Section 4.

\section{Minimal Surfaces with Free Boundaries on a Tetrahedron}

In comparison with polygonal boundaries, free or semifree boundaries raise new problems. In the polygonal case the function $D(s)$ tends towards infinity if $s \rightarrow \partial \mathcal{~}$. Therefore, it is possible to prove the existence of global minimizers and a mountainpass lemma, which guarantees the existence of an unstable solution if two different stable solutions exist. In the case of free boundaries the function $D(s)$ can be bounded on $\mathcal{U}$, so it is not possible to generalize all results from the polygonal case.

The simplest polyhedron example is a minimal surface in a tetrahedron; see the middle panel in Figure 4, left. The existence of such surfaces was shown for general tetrahedra in $\mathbb{R}^{3}$ by Smyth [1984] (who also proved uniqueness), for particular tetrahedra in $S^{3}$ by Karcher, Pinkall, and Sterling [Karcher et al. 1988], and again for particular tetrahedra in $H^{3}$ by Polthier [1989].

All these proofs were based on a conjugate surface construction, which is more complicated in the non-Euclidean case. An easier and more general proof of existence can be obtained by analyzing Shiffman's function, as shown in Figure 4, right. For $s \rightarrow \partial U$ the function $D(s)$ tends towards zero because the corresponding quasi-minimal surfaces degenerate. This was proved in [Nowak 1993; 1994] for any tetrahedron in any space form, and it is illustrated in the first of third panels of Figure 4. It follows that $D(s)$ has a maximum, which proves the existence of a minimal surface.

In the cases $c=0,1,-1$, Shiffman's function has the general shape shown in Figure 4, leading to the 

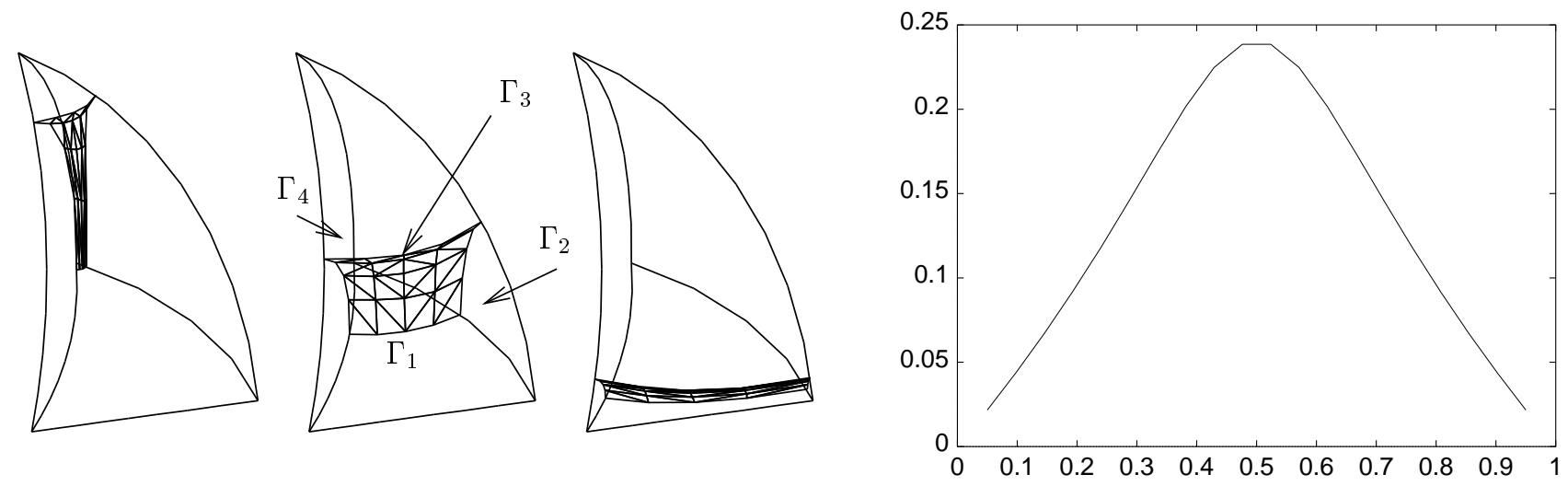

FIGURE 4. Quasi-minimal surfaces in a tetrahedron in $S^{3}$ and Shiffman's function.
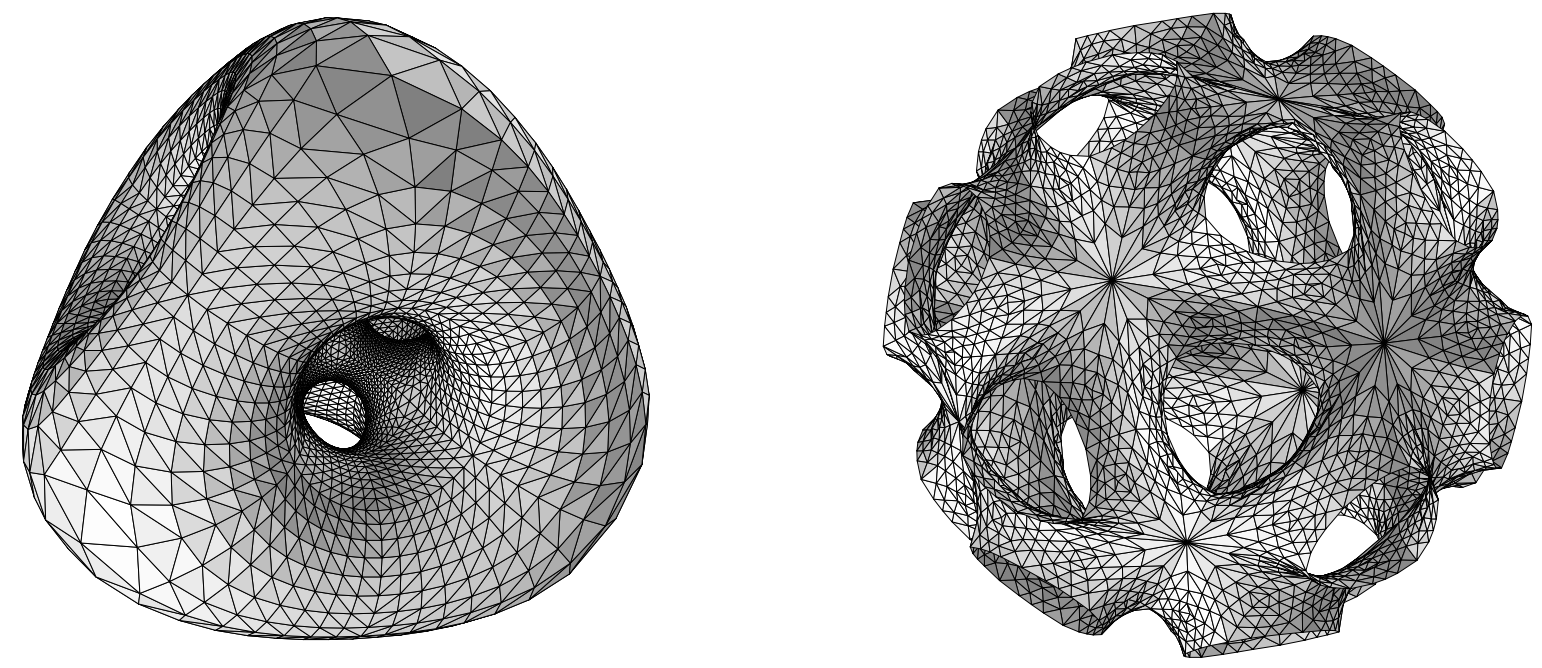

FIGURE 5. Left: Complete minimal surface in $S^{3}$ of Karcher, Pinkall, and Sterling. Right: Part of a complete minimal surface in $H^{3}$ of Polthier. The surfaces are shown in conformal projection.

conjecture that the surfaces are unique and have Morse index one. To my knowledge there are no rigorous results on the Morse index of minimal surfaces with free boundaries on a polyhedron.

Figure 5 shows the complete minimal surfaces of [Karcher et al. 1988] and [Polthier 1989]; as in Figure 3, these images were obtained by using the algorithm of Section 4 to construct generating patches as in Figure 4, then reflecting the patches.

\section{A Two-Parameter Minimal Surface in a Tetrahedron}

That the minimal surfaces discovered by Smyth are not the only ones in a tetrahedron is shown by the example in Figure 6, where the big tetrahedron is

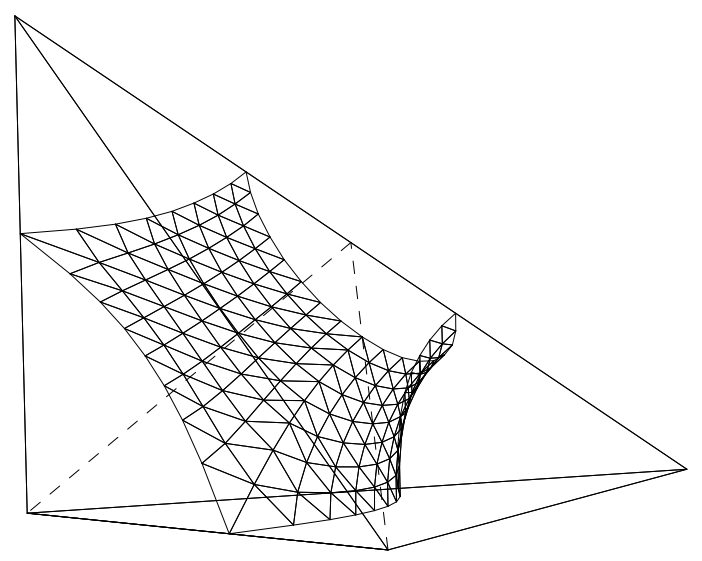

FIGURE 6. Minimal surface with five vertices on a tetrahedron. 

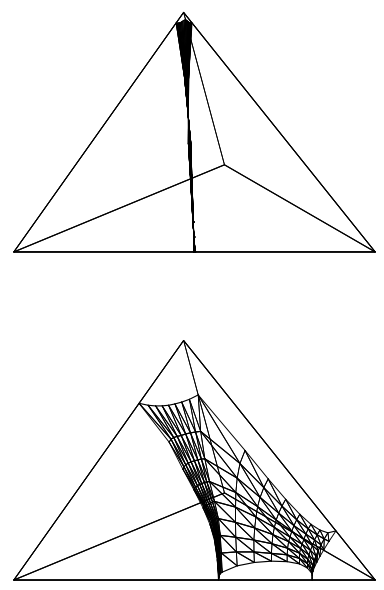
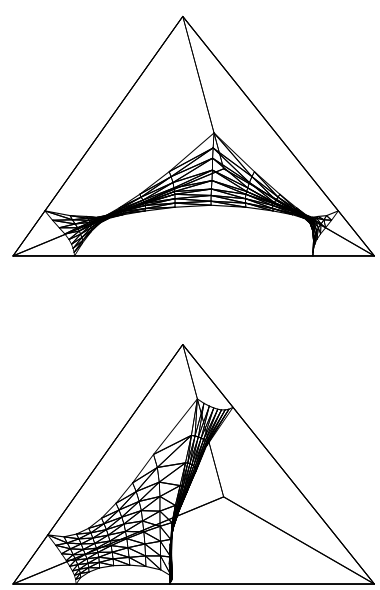

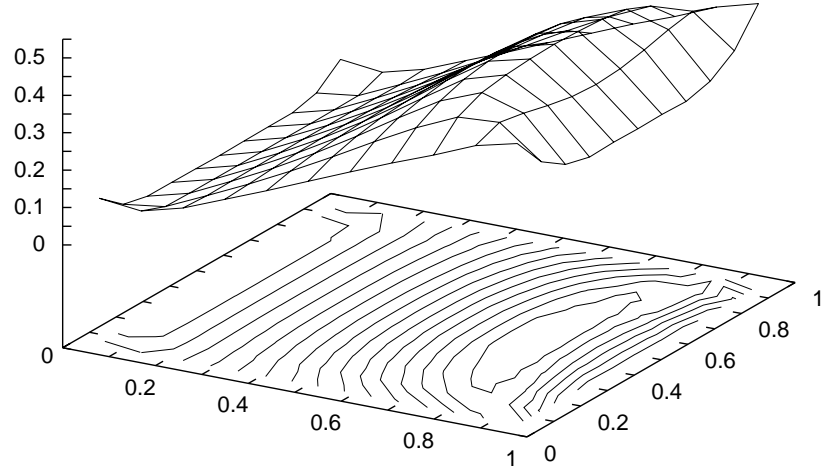

FIGURE 7. Left: Quasi-minimal surfaces for the configuration of Figure 6, as $s$ approaches $\partial \mathcal{U}$. Right: the corresponding Shiffman function.

symmetric with respect to a bisecting plane. The Smyth minimal surface patch belonging to one of the halves is reflected in the symmetry plane to yield a minimal surface with five vertices in the big tetrahedron. If we regard this as a Schwarzian chain problem for a five-edged surface (with two nonadjacent edges lying on the same plane), we get a two-dimensional parameter $s$. Figure 7, right, indicates that $D(s)$ has a unique maximum point, and hence that this minimal surface is unique and has Morse index two. Figure 7 indicates also that the corresponding quasi-minimal surfaces degenerate along only one parameter direction.

\section{Nonexistence of a Minimal Surface with Several Holes}

Figure 8 shows a quasi-minimal surface with three holes on the face of a regular tetrahedron. The surface was parametrized by the parameter domain of Figure 1, right. Since the tetrahedron is symmetric, the surface could also be constructed by six reflections of a surface patch in a subtetrahedron similar to that of Figure 6, having two orthogonal faces.

Numerical experiments show that, as the angle between the lower face and an adjacent face of the tetrahedron in Figure 6 is increased, the maximum point of Shiffman's function in Figure 7 tends toward $\partial \mathcal{U}$. It disappears if the two faces meet orthogonally. This leads to the conjecture that minimal surfaces with three holes similar as in Figure 8 do not exist. Minimal surfaces with several holes on the faces of a cube had been discovered by Schwarz; see [Dierkes et al. 1992].

\section{A New Minimal Surface and a Simple Example of Bifurcation}

The next example will show that Shiffman's variational principle is suitable for finding new solutions and studying bifurcation phenomena.

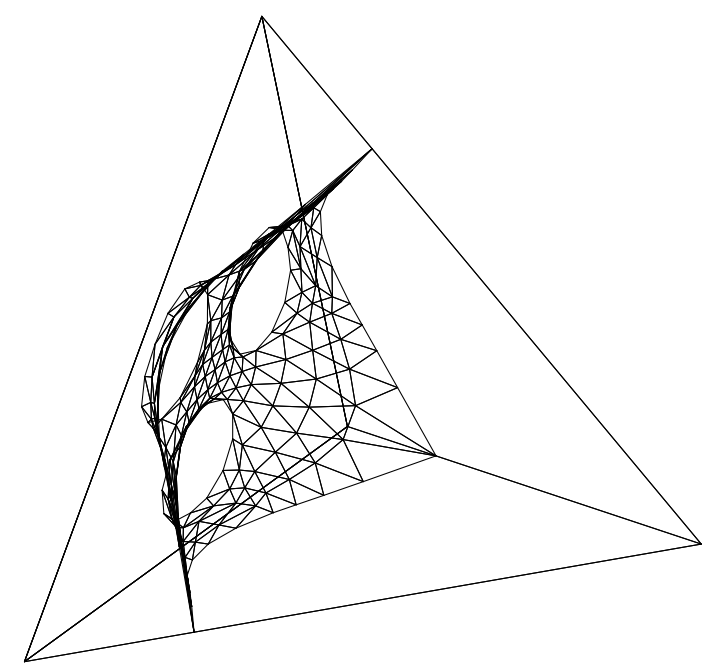

FIGURE 8. Quasi-minimal surface with three holes. 

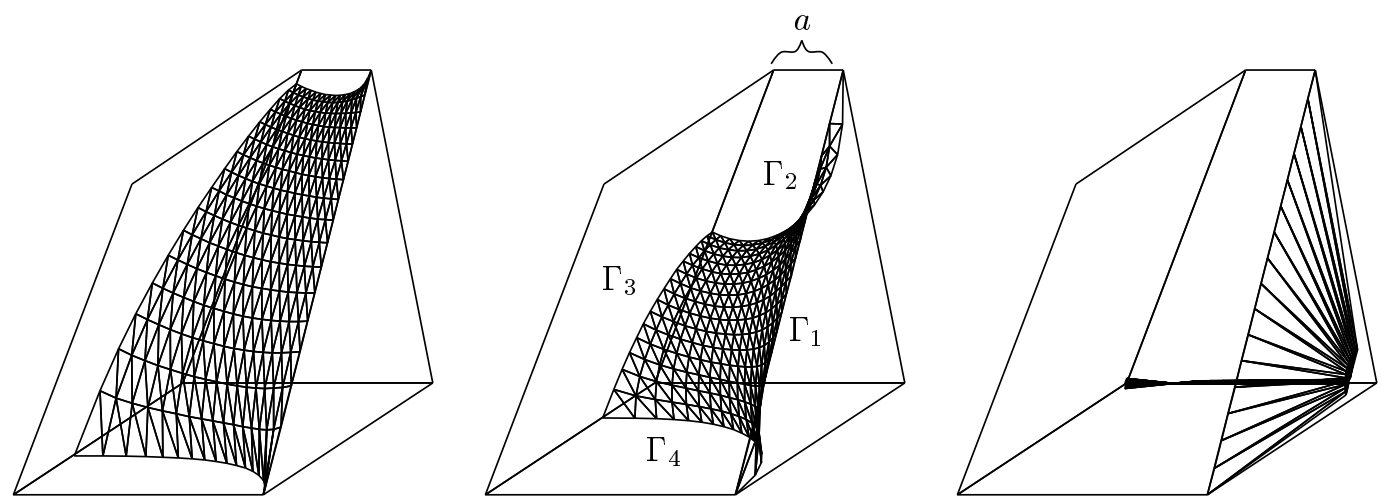

FIGURE 9. Stable, unstable, and degenerate minimal surfaces.

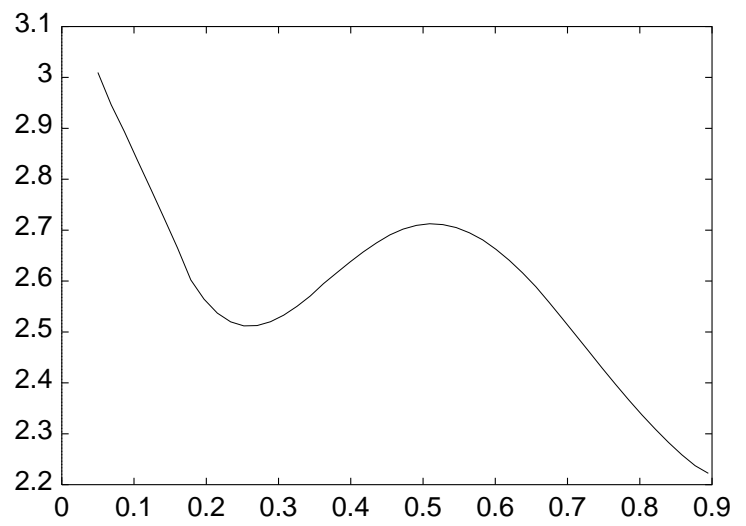

FIGURE 10. Shiffman's function for the example shown in Figure 9.

Figure 9 shows three minimal surfaces spanning a partly free Schwarzian chain, consisting of one line and three planes. The corresponding Shiffman function appears in Figure 10; the minimum corresponds to the first surface, which is therefore stable, whereas the maximum corresponds to the second, which is unstable. The third surface in Figure 9 is degenerate.

By reflection of these surfaces one obtains the triply connected polygonal surfaces of Figure 11.

Conjugation of the unstable solution (Figure 9, middle) leads to a stable one. This surface might be computed with the conjugation algorithm in [Pinkall and Polthier 1993]. However, this is not the case for the (locally) stable solution of (Figure 9, left). This example demonstrates the advantage of the quasi-minimal surface approach. Areaminimizing methods would lead to the degenerate solution, and other numerical methods that produce unstable minimal surfaces by direct approximation of critical points (such as the minimization of the squared mean curvature functional with Brakke's Evolver, as in [Hsu et al. 1992]) require a priori knowledge of a good initial surface. To our
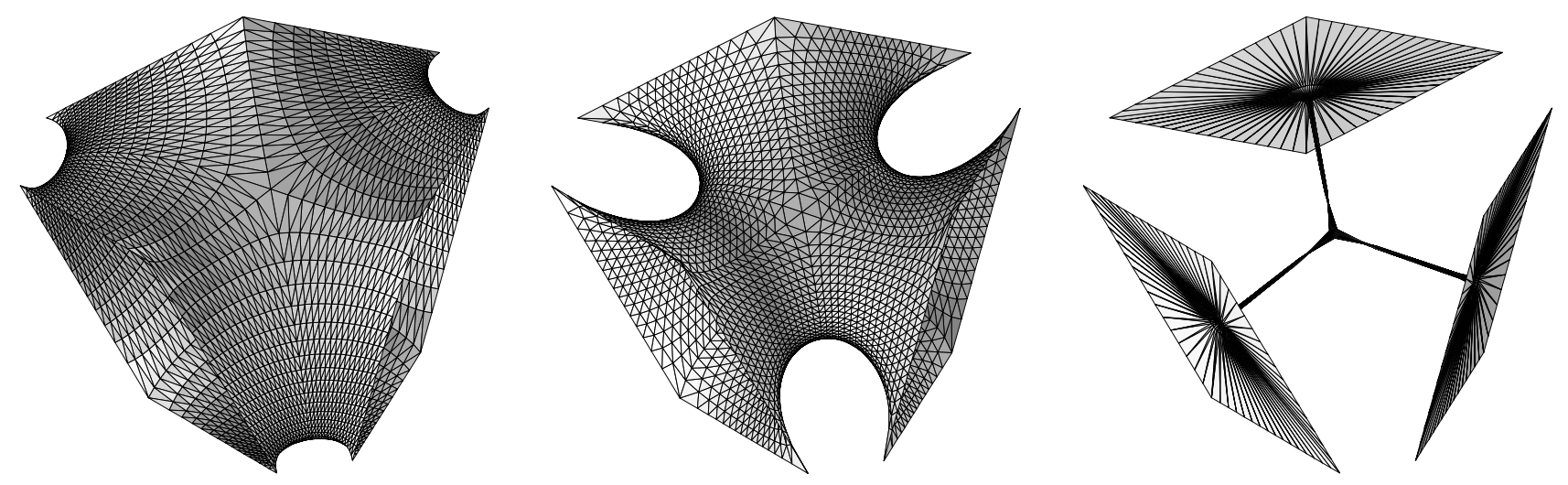

FIGURE 11. Triply connected polygonal minimal surfaces. 

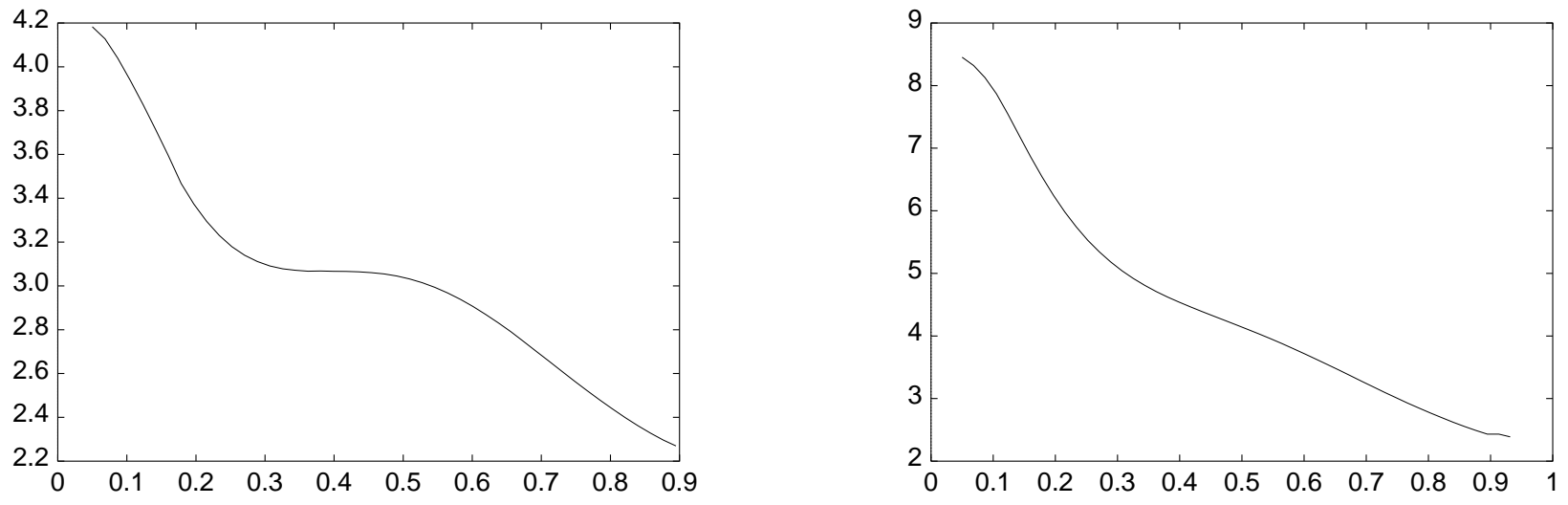

FIGURE 12. Shiffman's function for deformations of the example of Figure 9.

knowledge the stable surface in Figure 9 is new. It would be interesting to prove its existence rigorously. As we will now see, the existence depends on the geometry of the boundary configuration.

We will study deformations of the Schwarzian chain of Figure 9 by increasing the distance $a$ between the one-dimensional boundary piece and the opposite plane. As we saw, the Shiffman function for the situation of Figure 9 has two critical points (Figure 11). As a increases, these critical points approach, coalesce, and vanish, as shown in Figure 12. This is an example of a bifurcation, corresponding to a soap film that tears apart if the boundary is deformed too strongly. A similar phenomenon occurs with annular minimal surfaces spanning two parallel wire loops: if the loops are moved too far apart, the surface breaks up. In [Dierkes et al. 1992] the nonexistence of minimal surfaces in the latter case was shown by using the maximum principle for subharmonic functions.

\section{Minimal Surfaces in Nonconvex Polyhedra}

The existence of minimal surfaces in polyhedra has been proved only for certain convex polyhedra [Jost 1988]. Clearly there are minimal surfaces in nonconvex polyhedra as well, such as the surface of Figure 13, constructed by taking Smyth's solution in one of the three constituent tetrahedra (which has a dihedral angle of $120^{\circ}$ ) and reflecting twice. One imagines that nonconvex polyhedra obtained

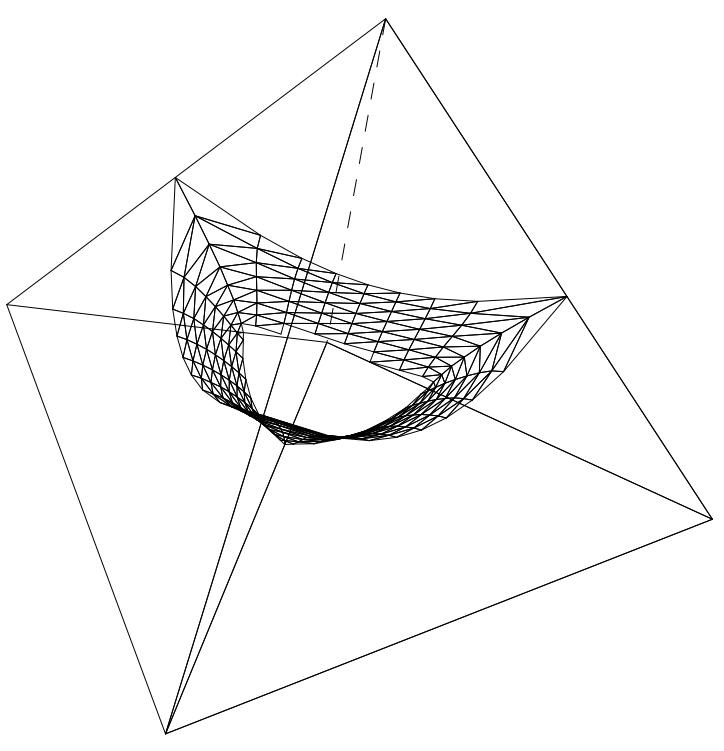

FIGURE 13. Minimal surface in a nonconvex polyhedron.

by small deformations of the symmetric polyhedron in Figure 13 also contain a minimal surface.

\section{Overshooting Minimal Surfaces}

An interesting unstable minimal surface is shown in Figure 14, left. The corresponding Schwarzian chain is a polygon with three straight lines and two planes that intersect at an angle of $30^{\circ}$. This example does not correspond to a physically viable minimal surface, but two remarks can be made.

First, the edge of the surface that is constrained to lie on $\Gamma_{4}$ overshoots the intersection of the two planes, and thus crosses $\Gamma_{5}$. The $\Gamma_{5}$-edge behaves 

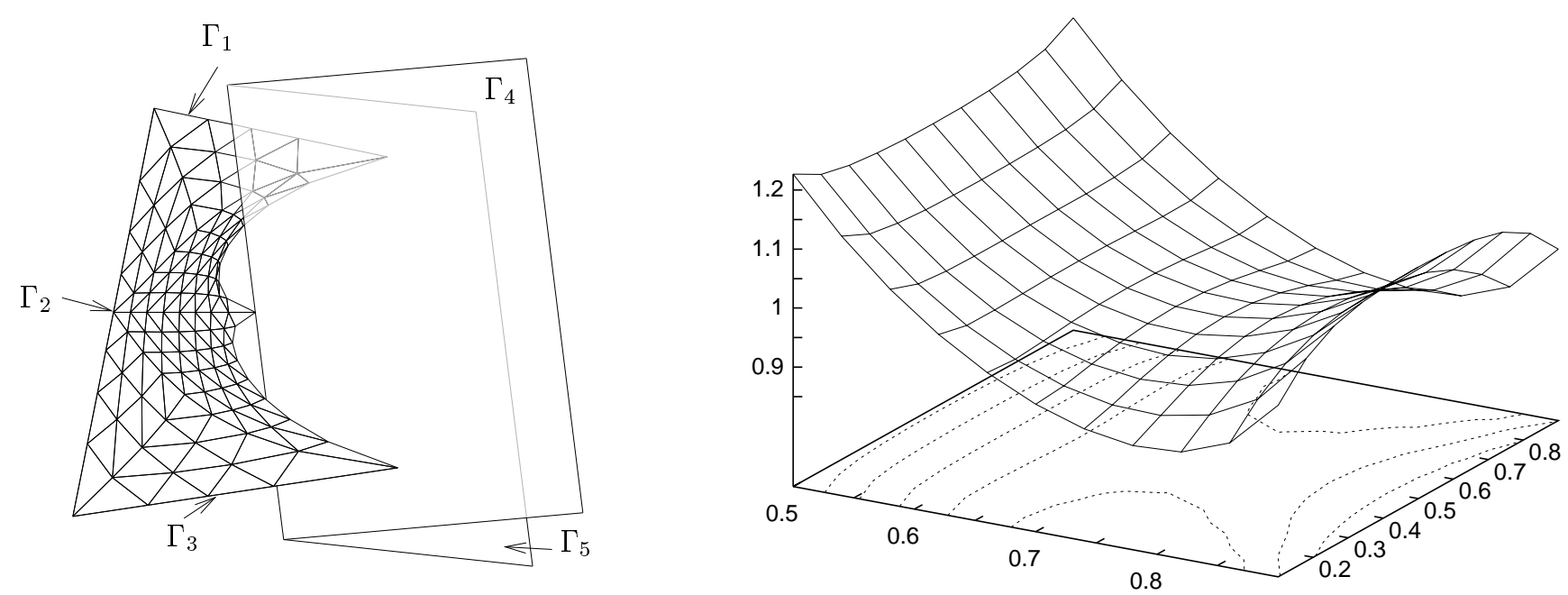

FIGURE 14. An unphysical minimal surface and the corresponding Shiffman function.

analogously. (It is an open question whether overshooting at the vertices can arise in the case of polygonal minimal surfaces; it has only been observed in the case of polygonal quasi-minimal surfaces [Lewerenz 1980/81].)

Secondly, the physically realistic problem could be solved by imposing additional inequality constraints guaranteeing that the free trace curve does not leave the half-planes. It can be assumed that the solution of the physically realistic problem has weaker regularity properties than Shiffman's variational problem. Recent investigations of S. Hildebrandt and F. Sauvigny suggest that the surface might creep along the singular intersection of the two planes. Here I would like to thank F. Sauvigny for interesting discussions.

\section{THE NUMERICAL METHOD}

\section{Discrete Variational Principle}

In this section we describe the numerical method implemented in [Nowak 1994]. In order to define discrete solutions of Problem B (page 305) we discretized Shiffman's function space $\mathcal{C}(\Gamma, I, s)$ by finite (linear triangle) elements. For this we defined for each parameter domain a starting triangulation and a refinement strategy (by partitioning interior triangles into four congruent ones and lifting boundary nodes onto $\partial \Omega_{s}$ ). In [Hinze 1994; Nowak 1994] it was shown that this refinement strategy leads in the case of convex or concave boundaries to quasi-uniform triangulations of $\Omega_{s}$; that is, the angles of the triangles do not degenerate if the grid size $h$ tends to zero. Triangulations of $\Omega_{s}$, which are denoted by $\Omega_{s}^{h}$, were shown in Figure 1 . The corresponding set of vertices is denoted by $P_{s}^{h}$. The triangulation is defined so that

$$
V_{s}:=\left\{\bar{\gamma}_{i}^{s} \cap \bar{\gamma}_{j}^{s}: i \neq j, 1 \leq i, j \leq l\right\} \subset P_{s}^{h} .
$$

The finite element space is denoted by $\mathcal{S}_{s}^{h}$; it consists of maps $X \in C^{0}\left(\bar{\Omega}_{s}^{h}, \mathbb{R}^{n}\right)$ such that $\left.X\right|_{T}$ is linear for all triangles $T$ of $\Omega^{h}$, where $n=3$ if $c=0$ and $n=4$ if $c \neq 0$. The discrete Shiffman function space is denoted by

$$
\mathrm{e}^{h}(\Gamma, I, s) \text {; }
$$

it consists of all $Y \in \mathcal{S}_{s}^{h}$ such that

$$
Y(z) \in M(c) \quad \text { if } z \in P_{s}^{h}
$$

and $X(z) \in \Gamma_{I(i)}$ if $z \in P_{s}^{h} \cap \bar{\gamma}_{i}^{s}$, for $1 \leq i \leq l$.

The discrete version of Problem $B$ is this:

Problem C. Minimize $D_{\Omega_{s}^{h}}(Y)$ subject to the condition $Y \in \mathrm{C}^{h}(\Gamma, I, s)$, where $s \in \mathcal{U}^{\prime}$ minimizes $C_{h}(s)$.

Here the discrete Dirichlet integral $D_{\Omega_{s}^{h}}(Y)$ is defined in the same manner as in the continuous case 
(although $Y$ does not map to $M(c)) ; C_{h}(s)$ is defined in the same manner as $C(s)$; and $\mathcal{U}^{\prime} \Subset \mathcal{U}$ is a neighborhood of an optimal parameter $s^{*}$.

The minimization of $D_{\Omega_{s}^{h}}(Y)$ is a nonlinear optimization problem with a quadratic objective function and quadratic constraints. In [Nowak 1994] it was proved that a solution exists and is unique if $c \geq 0$. (For $c<0$ the uniqueness is an open problem.) The optimization problem is solved iteratively by determining the minimum of $D_{\Omega_{s}^{h}}(\cdot)$ on the tangent space of $\mathrm{C}^{h}(\Gamma, I, s)$ with respect to an approximation $X_{h}^{(k)}$. Projecting this solution onto $\mathrm{C}^{h}(\Gamma, I, s)$ one gets a better approximation $X_{h}^{(k+1)}$. In [Nowak 1994] it was proved, using [Spellucci 1993, Theorem 3.4.1], that this iteration converges towards the desired solution.

It turns out that the convergence of this method is not much slower when $c>0$ than when $c=0$. If $c<0$ the convergence is slower. In this case the discrete energy functional is not necessarily convex. (This was shown in [Nowak 1994] by giving a vector $\Psi$ in the tangent space of $\mathrm{C}^{h}(\Gamma, I, s)$ such that $\delta^{2} D_{\Omega_{s}^{h}}(X, \Psi)=D_{\Omega_{s}^{h}}(\Psi)<0$.) We assume that the use of a conformal model of $H^{3}$ in $\mathbb{R}^{3}$ would bring better results in the case $c<0$.

The search for optimal $s$ was performed using standard unconstrained optimization techniques; see [Press et al. 1986], for example.

\section{Convergence Results}

In order to prove the convergence of discrete minimal surfaces defined by Problem $\mathrm{C}$ towards a solution of problem $\mathrm{B}$, it is necessary to show that discrete quasi-minimal surfaces converge uniformly on given subsets $\mathcal{U}^{\prime} \Subset \mathcal{U}$. This can be done with the aid of the following convergence result established for disk-type polygonal surfaces in $\mathbb{R}^{3}$ [Hinze 1994]:

$$
\max _{s \in \mathcal{U}^{\prime}}\left\|X_{h}(s)-X(s)\right\|_{1, B_{s}^{h}} \leq C \cdot h^{\mu}, \quad \mu>0 .
$$

Here $\mu$ depends on the smallest angle of the polygon, and $B_{s}^{h}$ denotes a triangulation of the disk $B_{s}$.
The result (4-1) could be obtained because the analyticity of disk-type polygonal quasi-minimal surfaces $X(z, s)$ on $B_{s} \backslash V_{s} \times \mathcal{U}$ and asymptotic expansions of $X(z, s)$ in $V_{s}$ were proved in [Heinz 1979]. Using (4-1) in [Nowak 1994] it was shown for polygonal disk-type surfaces in $\mathbb{R}^{3}$ (that is, for $c=0)$ that $\left|C_{h}(s)-C(s)\right|_{\infty, u^{\prime}} \rightarrow 0$. This yields $s_{h}^{*} \rightarrow s^{*}$ and

$$
\left\|X_{h}\left(s_{h}^{*}\right)-X\left(s^{*}\right)\right\|_{1, B^{h}} \rightarrow 0 .
$$

For the general case $(c \neq 0)$ we derived convergence only for quasi-minimal surfaces:

$$
\left\|X_{h}(s)-X(s)\right\|_{1, \Omega} \rightarrow 0 .
$$

(For the sake of simplicity we assume $\Omega^{h}=\Omega$.)

We give a short outline of the proof, which consists of two parts.

In the first part the uniform boundedness of the sequence $\left\{X_{h}(s)\right\}_{h \rightarrow 0}$ in $H^{1}(\Omega)$ is shown by using a Poincaré inequality. This implies that a subsequence converges weakly in $H^{1}(\Omega)$ and therefore strongly in $L^{2}(\Omega)$ to a surface $\bar{X}$.

In the second part of the proof it is shown that $\bar{X} \in \mathcal{C}(\Gamma, I, s)$. Because of Rellich's theorem, $\bar{X}$ fulfills the boundary conditions. Therefore, it is sufficient to show that $|\bar{X}|_{c}^{2}=1 / c$ almost everywhere in $\Omega$; that is, that $\left.|| \bar{X}\right|_{c} ^{2}-\left.\frac{1}{c}\right|_{0, \Omega}=0$.

Let $\omega_{i}$ denote the polygonal domains that are defined by the centers of the triangles meeting in one point $p_{i}$ corresponding to a triangulation of $\Omega$, i.e., $\bigcup \omega_{i}=\Omega$. The operator $\Pi_{h}$ is defined by $\Pi_{h} x=p_{i}$ for all $x \in \omega_{i}$. Therefore $\left|X \circ \Pi_{h}\right|_{0, \Omega}$ is a Riemann sum of a function $X$ and converges towards $|X|_{0, \Omega}$ for $h \rightarrow 0$. We estimate, using the equality $\left|X_{h}(s)\left(p_{i}\right)\right|_{c}^{2}=1 / c$ :

$$
\begin{aligned}
\left.|| \bar{X}\right|_{c} ^{2}-1 /\left.c\right|_{0, \Omega}=\left.|| \bar{X}\right|_{c} ^{2}-\left.\left|X_{h}(s)\right|_{c}^{2} \circ \Pi_{h}\right|_{0, \Omega} \\
\leq\left|\left(|\bar{X}|_{c}^{2}-\left|X_{h}(s)\right|_{c}^{2}\right) \circ \Pi_{h}\right|_{0, \Omega} \\
\quad+\left.|| \bar{X}\right|_{c} ^{2}-\left.|\bar{X}|_{c}^{2} \circ \Pi_{h}\right|_{0, \Omega} .
\end{aligned}
$$

The right-hand side of this inequality converges to zero, because $\left|X_{h}(s)\right|_{c}^{2} \rightarrow|\bar{X}|_{c}^{2}$ almost everywhere 
on $\Omega$ for $h \rightarrow 0$ and because of the property of the operator $\Pi_{h}$.

By the lower semicontinuity of Dirichlet's functional it follows $D_{\Omega}(\bar{X})=D_{\Omega}(X(s))$ and therefore $\bar{X}=X(s)$ by uniqueness of $X(s)$. This implies $(4-2)$.

We mention that it would be highly desirable to generalize the results of [Heinz 1979] to the nonEuclidean situation. This would lay the foundation of a general convergence proof.

\section{REFERENCES}

[Brakke 1992] K. A. Brakke, "The Surface Evolver", Experiment. Math. 1:2 (1992), 141-165.

[Chopp and Sethian 1993] D. L. Chopp and J. A. Sethian, "Flow under curvature: singularity formation, minimal surfaces, and geodesics", Experiment. Math. 2:4 (1993), 235-255.

[Dierkes et al. 1992] U. Dierkes, S. Hildebrandt, A. Küster, and O. Wohlrab, Minimal surfaces I: Boundary value problems, Grundlehren der Mathematischen Wissenschaften 295, Springer, Berlin, 1992.

[Dziuk and Hutchinson 1996] G. Dziuk and J. E. Hutchinson, "On the approximation of unstable parametric minimal surfaces", Calc. Var. Partial Differential Equations 4:1 (1996), 27-58.

[Heinz 1979] E. Heinz, "Über die analytische Abhängigkeit der Lösungen eines linearen elliptischen Randwertproblems von Parametern", Nachr. Akad. Wiss. Göttingen II Math.-Phys. Kl.no. 1 (1979), 1-12.

[Hinze 1994] M. Hinze, On the numerical treatment of minimal surfaces with polygonal boundaries, Dissertation, Technische Universität Berlin, 1994.

[Hsu et al. 1992] L. Hsu, R. Kusner, and J. Sullivan, "Minimizing the squared mean curvature integral for surfaces in space forms", Experiment. Math. 1:3 (1992), 191-207.

[Hutchinson 1991] J. E. Hutchinson, "Computing conformal maps and minimal surfaces", pp. 140-161 in Workshop on Theoretical and Numerical Aspects of Geometric Variational Problems (Canberra, 1990), edited by G. Dziuk et al., Proc. Centre Math. Appl. Austral. Nat. Univ. 26, Austral. Nat. Univ., Canberra, 1991.
[Jarausch 1978] H. Jarausch, Zur numerischen Behandlung von parametrischen Minimalflüchen mit finiten Elementen, Dissertation, Univ. Bochum, 1978.

[Jost 1988] J. Jost, "Embedded minimal disks with a free boundary on a polyhedron in $\mathbb{R}^{3}$, Math. Z. 199:3 (1988), 311-320.

[Jost 1989] J. Jost, "Das Existenzproblem für Minimalflächen", Jahresber. Deutsch. Math.-Verein. 91:1 (1989), 1-32.

[Jost 1991] J. Jost, Two-dimensional geometric variational problems, Pure and Applied Mathematics, John Wiley, Chichester, 1991.

[Karcher et al. 1988] H. Karcher, U. Pinkall, and I. Sterling, "New minimal surfaces in $S^{3}$ ", J. Differential Geom. 28:2 (1988), 169-185.

[Lawson 1970] H. B. Lawson, Jr., "Complete minimal surfaces in $S^{3 "}$, Ann. of Math. (2) 92 (1970), 335374.

[Lewerenz 1980/81] F. Lewerenz, "Eine Bemerkung zu den Marx-Shiffmanschen Minimalvektoren bei Polygonen", Arch. Rational Mech. Anal. 75:2 (1980/81), 199-202.

[Nowak 1993] I. Nowak, "Minimal surfaces with prescribed topological type on a Schwarzian chain in $M_{c}^{3} "$, Ann. Global Anal. Geom. 11:4 (1993), 331344.

[Nowak 1994] I. Nowak, Approximation von Minimalflüchen mit vorgeschriebenem topologischem Typ auf Schwarzschen Ketten in $M^{3}(c)$, Dissertation, Technische Universität Berlin, 1994.

[Pinkall and Polthier 1993] U. Pinkall and K. Polthier, "Computing discrete minimal surfaces and their conjugates", Experiment. Math. 2:1 (1993), 15-36.

[Polthier 1989] K. Polthier, Neue Minimalflächen in $H^{3}$, Diplomarbeit, Universität Bonn, 1989.

[Press et al. 1986] W. H. Press, B. P. Flannery, S. A. Teukolsky, and W. T. Vetterling, Numerical recipes: The art of scientific computing, Cambridge University Press, Cambridge, 1986.

[Radó 1930] T. Radó, "Some remarks on the problem of Plateau", Proc. Natl. Acad. Sci. USA 16 (1930), 242-248. 
[Sauvigny 1985a] F. Sauvigny, "Ein Eindeutigkeitssatz für Minimalflächen in $\mathbb{R}^{p}$ mit polygonalem Rand", $J$. Reine Angew. Math. 358 (1985), 92-96.

[Sauvigny 1985b] F. Sauvigny, "On the Morse index of minimal surfaces in $\mathbb{R}^{p}$ with polygonal boundaries", Manuscripta Math. 53:3 (1985), 167-197.

[Smyth 1984] B. Smyth, "Stationary minimal surfaces with boundary on a simplex", Invent. Math. 76:3 (1984), 411-420.

[Spellucci 1993] P. Spellucci, Numerische Verfahren der nichtlinearen Optimierung, Internationale Schriftenreihe zur Numerischen Mathematik, Birkhäuser, Basel, 1993.
[Ströhmer 1980] G. Ströhmer, "Instabile Minimalflächen in Riemannschen Mannigfaltigkeiten nichtpositiver Schnittkrümmung", J. Reine Angew. Math. 315 (1980), 16-39.

[Tsuchiya 1987] T. Tsuchiya, "Discrete solution of the Plateau problem and its convergence", Math. Comp. 49:179 (1987), 157-165.

[Wente 1971] H. C. Wente, "A general existence theorem for surfaces of constant mean curvature.", Math. Z. 120 (1971), 277-288.

[Wohlrab 1995] O. Wohlrab, Zur numerischen Behandlung von parametrischen Minimalflächen mit halbfreien Rändern, Dissertation, Universität Bonn, 1995.

Ivo Nowak, Institut für Mathematik der Brandenburgischen Technischen Universität Cottbus, Karl-Marx-Straße 12, D-03044 Cottbus, Germany (ivo@math.tu-cottbus.de)

Received January 11, 1995; accepted in revised form January 15, 1997 